\title{
Finite Difference Scheme for Planar Waveguides with Arbitrary Index Profiles and its Implementation for Anisotropic Waveguides with Diagonal Permitivity Tensor
}

\author{
H. P. Uranus ${ }^{1,2}$, H.J.W.M. Hoekstra ${ }^{1}$, E.W.C van Groesen ${ }^{2}$ \\ ${ }^{1}$ Dept. of Applied Physics and ${ }^{2}$ Dept. of Mathematical Sciences \\ University of Twente \\ P.O. Box 217, 7500 AE Enschede, The Netherlands \\ e-mail: h.p.uranus@el.utwente.nl
}

\begin{abstract}
Finite difference scheme with uniform mesh for planar waveguides with arbitrary refractive index profiles that take full account of any smooth index variation and index discontinuity was derived for TE and TM-polarized waves. Second-order accurate discretizations for TE, firstand second-order accurate for TM at index discontinuities around interfaces between segments of different index profiles are presented. At the computational boundaries, transparent boundary conditions have been used. The scheme was implemented for anisotropic waveguides with diagonal permitivity tensor and examined by using samples with various refractive index profiles, ranging from simple step- and graded-index up to complicated refractive index profile waveguide structures composed of either isotropic or anisotropic materials. For simple cases where the results of other methods are available in the literature, the proposed scheme shows very good agreement.
\end{abstract}

Keywords: finite difference, interface correction, arbitrary index, anisotropic waveguide, discretization 